Original Research Article

\title{
Cross sectional study on prevalence and medication adherence of hypertension and diabetes in a tertiary care hospital in Karimnagar, India
}

\author{
S. Sre Akshaya Kalyani*, Srihitha Pendota, Abhinay Sharma Katnapally, \\ Dharanija Porandla, Sandeep Bheemreddy
}

\begin{abstract}
Department of Clinical Pharmacy, Vaageswari College of Pharmacy, Karimnagar, Telanagana, India
\end{abstract}

Received: 23 January 2018 Accepted: 05 March 2018

*Correspondence to: Dr. S. Sre Akshaya Kalyani, Email: akshaya273@gmail.com

Copyright: (C) the author(s), publisher and licensee Medip Academy. This is an openaccess article distributed under the terms of the Creative Commons Attribution NonCommercial License, which permits unrestricted noncommercial use, distribution, and reproduction in any medium, provided the original work is properly cited.

\begin{abstract}
Background: The study aimed to assess the prevalence of Hypertension (HTN), Diabetes mellitus(DM) and other diseases along with comorbid conditions, disease complications and also to assess medication adherence in a tertiary hospital in Karimnagar, Telangana, India.

Methods: A Cross sectional study is performed by analysing a total of 500 patient's individual case safety reports (ICSR). Prevalence of Hypertension and Diabetes along with most prescribed drugs are analysed. Medication adherence is analysed by using Morisky Medication adherence questionnaire scale (MMAS4).

Results: Patients with past history of DM, HTN and other diseases which was found to be 245 (49\%) patients. Patients with highest disease prevalence were found to be with HTN $(56.73 \%)$, DM (31.83\%) followed by other diseases like asthma $(8.57 \%)$, Cerebrovascular accident $(4.89 \%)$ etc., Total of 66 Patients were ruled out with comorbid diseases. Patients with HTN+DM (47) were found to be highest followed by DM+HTN+CVA (4). MMAS-4 revealed 208 patients were using medication out of 245 patients with previous history. According to MMAS4 most of the patients were with medium adherence (76). Prevalent drug used for HTN include Amlodipine and for DM Telmisartan+Hydrochlorthiazide.

Conclusions: The study revealed that almost half of the admitted patients were with HTN and DM. The patients were counselled properly to adhere strictly to the prescription. Medication adherence to HTN and DM was found to be good in this study. Since the disease complications were also ruled out, the health care professionals are recommended to spread awareness on DM and HTN and disease management in order to control disease and improve health outcomes.
\end{abstract}

Keywords: Diabetes mellitus, Hypertension, Karimnagar, Medication adherence, Morisky Medication adherence questionnaire scale (MMAS-4), Prevalence of Hypertension and diabetes mellitus

\section{INTRODUCTION}

DM and HTN are most common and serious diseases with chronic complications and these both are risk factors for cardiovascular diseases which increases the risk of stroke, myocardial infarction, heart failure, and chronic kidney disease. ${ }^{1,2}$ The main objective of this study is to find out prevalence of both HTN and DM in hospitalized patients along with comparison of age and gender. Up to $75 \%$ of adults with diabetes also have HTN, and patients with HTN alone show insulin resistance. ${ }^{3,4}$ 
Data from many epidemiologic sources suggest that the prevalence of HTN in patients with DM is approximately 1.5-2.0 times greater than in non diabetic population. ${ }^{3}$ Thus, HTN and DM are common, interlinked diseases that share a significant underlying risk factors (including ethnicity, familial, dyslipidemia, and lifestyle determinants) and complications. ${ }^{5-7}$ Along with prevalence of HTN and DM, we also studied the complications of both the diseases. Different complications like Cerebrovascular accident (CVA), diabetic nephropathy (DN), Chronic kidney disease (CKD), Deep vein thrombosis (DVT) etc. are observed. ${ }^{8-10}$

One of the most important issue in the effective treatment of Sub chronic and chronic illnesses is patient medication adherence to prescribed medications. ${ }^{10-12}$

Adherence is the degree to which a person's behaviour coincides with medical advice. ${ }^{13-16}$ Adherence to prescribed medicines is a way to improve one's health, but patients may or may not adhere to the prescriptions. In this study medication adherence is measured using MMAS-4 questionnaire and details were noted. ${ }^{17-20}$

\section{METHODS}

A Prospective (Cross sectional) observational study was performed in a tertiary care hospital with 500 patients. Study Sampling involved patients with or without previous medical history who are hospitalized.

The factors that were took into consideration include Age and gender effect on disease onset and prolongation, Prevalence of diseases, Comorbid conditions, Medication adherence, Disease Complications, Prevalent drug prescribed to treat HTN and DM.

The time period of this study is from July 2017 to January 2018 which is of 7 months.

\section{Study population}

The participants of the study were taken from patients who were admitted to the in-patient setting of tertiary care hospital in Karimnagar. All the patients provided consent for the study. A total of 500 patients were recruited in the study.

\section{Inclusion criteria}

- $\quad$ Patients above age of 14 years

- All patients with past medical history i.e., DM, HTN, asthma, Thyroid etc.,

- Patients admitted with complications of the above diseases,

- Patients with multiple co-morbidities and those taking multiple medications for longer period of time,

- Only patients who are hospitalized during study period,
- $\quad$ Patients of both genders.

\section{Exclusion criteria}

- $\quad$ Patients of less than 14 years of age.

- $\quad$ Patients who are admitted in oncology department.

- Women who are admitted in gynaecology department.

- $\quad$ Patients with previous surgical history

- Patients with only any infectious disease previously like dengue, malaria etc.,

- $\quad$ Patients declining study participation. ${ }^{2}$

\section{Data collection}

Data were collected over a period of 7 months of patients with and without HTN and DM patients. Individual case safety reports of all the patients were observed and the following data was obtained from each patient:

1. Demographic details- age, sex

2. Complaints of admission and present illness, final diagnosis

3. Medical and medication history

4. Habits- type of diet, smoking, alcohol consumption

5. Laboratory findings- serum biochemistry, blood pressure.

6. Morisky medication adherence scale (MMAS-4) questionnaire

\section{Statistical analyse}

The results were given using XL sheet:

1. To stratify subjects according to presence or absence of past medical history

2. To find out the prevalence of both HTN and DM along with other diseases and complications like Asthma, Tuberculosis (TB), Hypothyroidism, Epilepsy, CVA, CKD, Chronic Obstructive Pulmonary disease (COPD), Diabetic nephropathy (DN), Myocardial infarction (MI), Chronic artery disease (CAD), Hyperthyroidism.

3. To find out the mean of Comorbid disease conditions like DM, HTN and vice-versa. DM, HTN with other complications like CVA, CKD, DN and DVT.

4. To find out percentage of disease complications

5. To check the percentage of medication adherence in the population

6. To analyze effect of gender on disease

7. To rule out the most prescribed drug for treating DM in the specific population

\section{RESULTS}

Of the total population the percentage with past medical history are $255(51 \%)$. Among which patients with HTN are $139(54.5 \%)$, DM patients-75 (29.41\%), Asthma patients-21 (8.23\%), TB-14 (5.49\%), Hypothyroidism-13 (5.09\%), CVA-12 (4.7\%), Epilepsy-11 (4.313\%), CKD-07 
(2.74\%), COPD-04 (1.56\%), DN-02 (0.78\%), MI-02 (0.78\%), CAD-01 (0.39\%), Hyperthyroidism-01 (0.39\%), Other diseases were present in $52(20.39 \%)$ patients.

Disease complications were observed in $85(33 \%)$ patients. The diseases with many complications include both HTN and DM with a percentage of $71.2 \%$. In some cases, HTN lead to DM, where in some other cases DM lead to HTN. Other complications observed include diabetic foot, Diabetic nephropathy, Acute kidney injury, Chronic kidney disease, CVA, Deep vein thrombus, asthma lead to COPD in some cases.

MMAS-4 questionnaire was assessed and 76(36.53\%) patients were found to be with Medium adherence(MA), $68(32.69 \%)$ patients with High adherence(HA), and 64 $(25.09 \%)$ patients with low adherence(LA). Disease complications were observed mostly in low adherence patients.

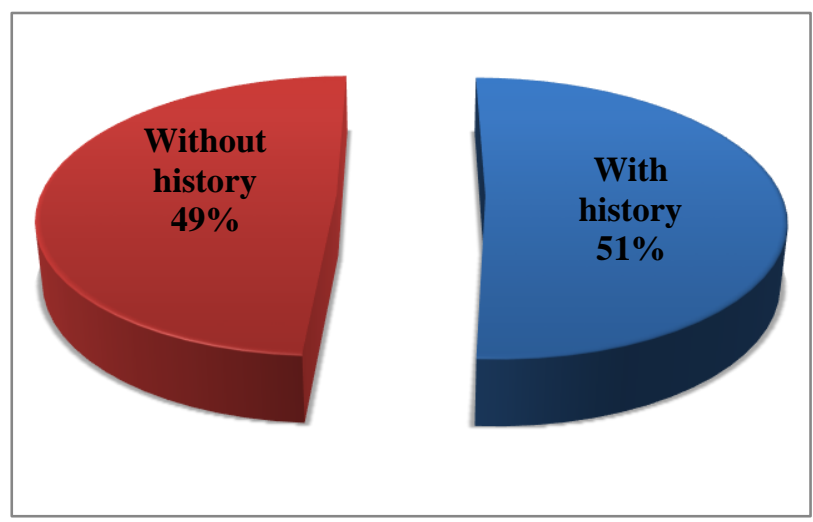

Figure 1: Percentage of population with or without medication history.

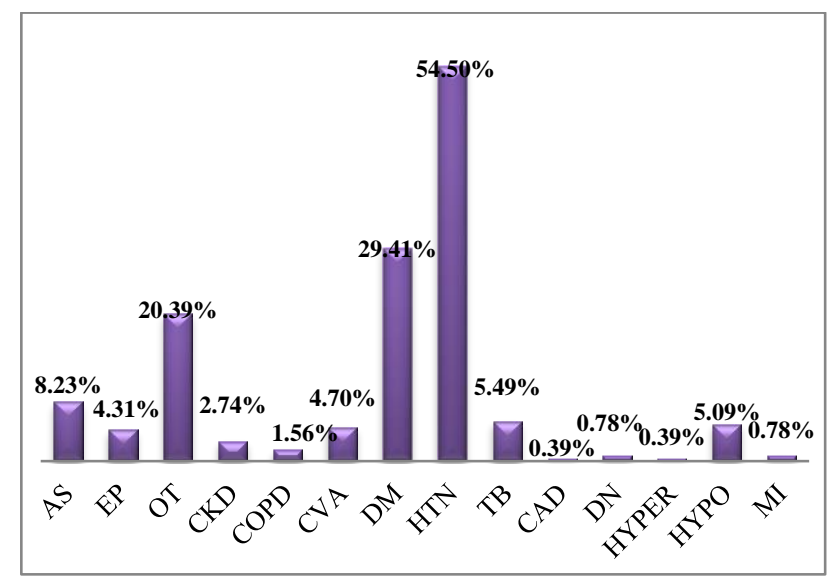

(AS $\rightarrow$ Asthma; $\quad$ EP $\rightarrow$ Epilepsy; $\quad$ OT $\rightarrow$ Other diseases; $\mathrm{CKD} \rightarrow$ Chronic kidney disease; COPD $\rightarrow$ Chronic obstructive pulmonary disease; CVA $\rightarrow$ Cerebrovascular accident; $D M \rightarrow$ Diabetes $\quad$ Mellitus; $\quad$ HTN $\rightarrow$ Hypertension; $\mathrm{TB} \rightarrow$ Tuberculosis; $\quad \mathrm{CAD} \rightarrow$ Chronic Artery Disease; DN $\rightarrow$ Diabetic nephropathy; Hyper $\rightarrow$ Hyperthyroidism; Hypo $\rightarrow$ Hypothyroidism; MI $\rightarrow$ Myocardial Infarction)

Figure 2: Prevalence of DM and HTN along with other diseases.

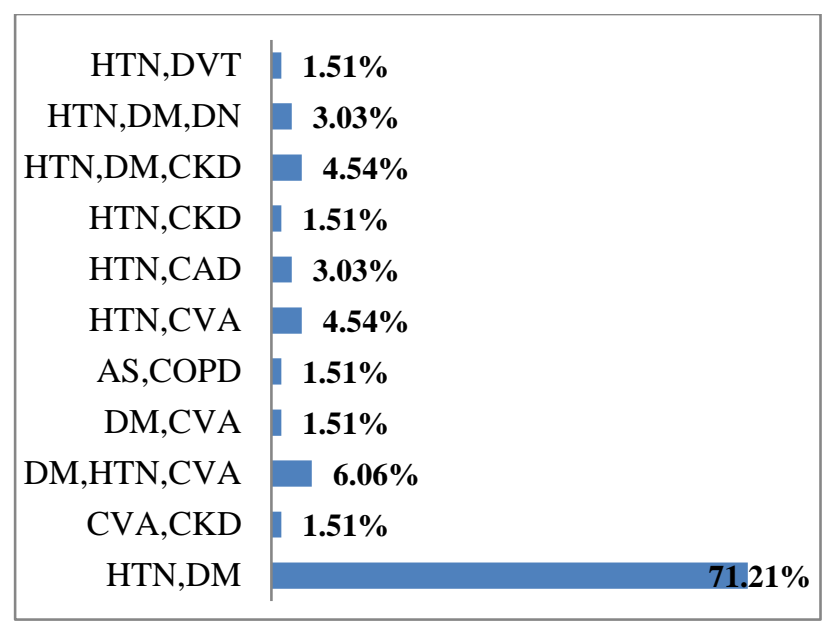

DVT $\rightarrow$ Deep Vein Thrombosis and Other abbreviations are already denoted.

Figure 3: Prevalence of comorbid diseases.

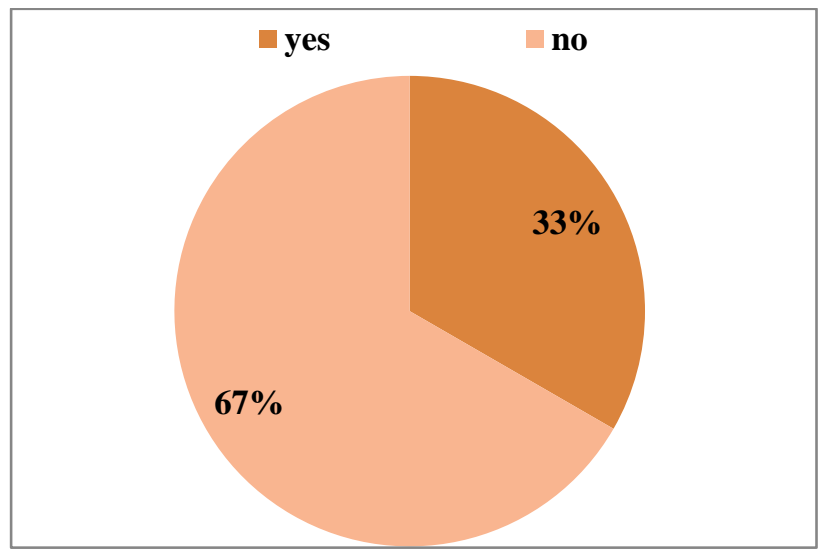

Yes $\rightarrow$ Patient population with disease complication.

No $\rightarrow$ Patient population without disease complication.

Figure 4: Percentage of complications of different diseases.

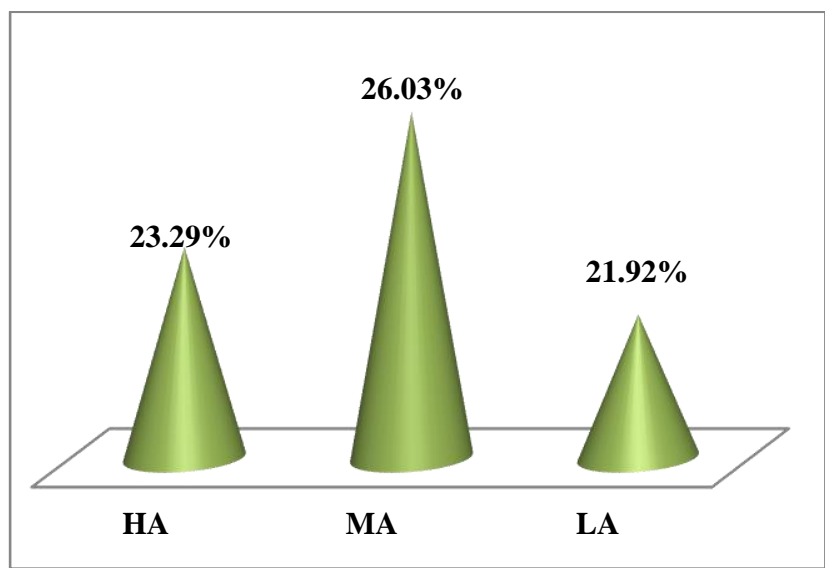

MMAS-4 $\rightarrow$ Morisky medication adherence scale

$\mathrm{HA} \rightarrow$ High adherence

$\mathrm{MA} \rightarrow$ Medium adherence

LA $\rightarrow$ Low adherence

Figure 5: Adherence output using Morisky Medication adherence scale. 


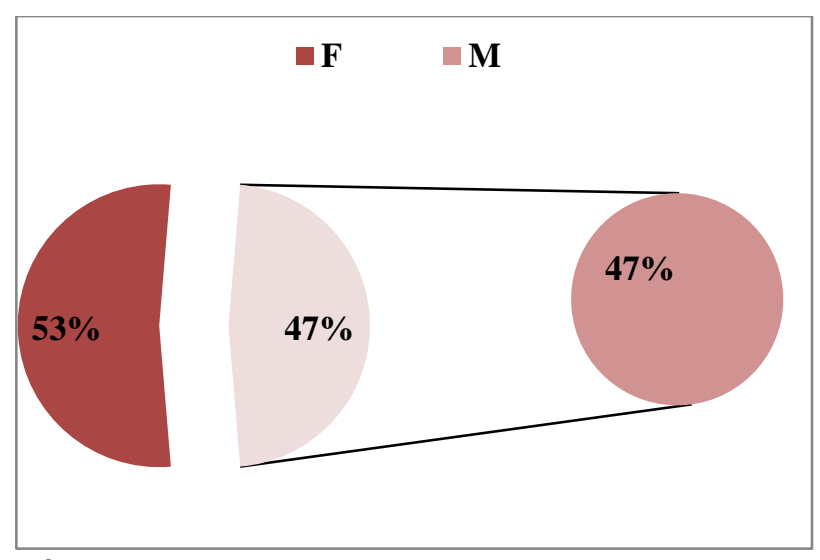

$\mathrm{F} \rightarrow$ Female $\mathrm{M} \rightarrow$ Male

Figure 6: Effect of DM and HTN on gender distribution.

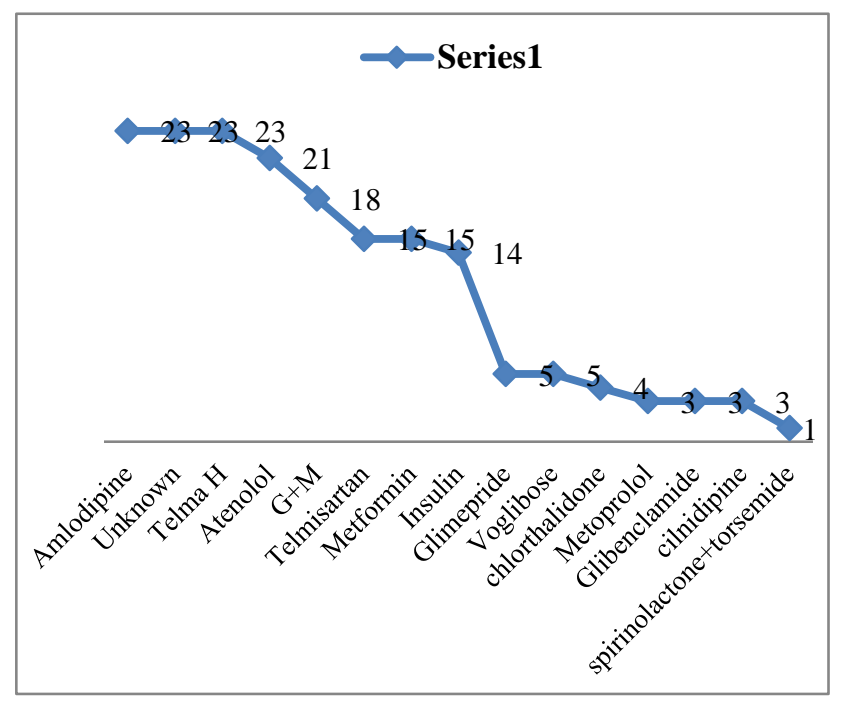

Figure 7: Prevalent drugs prescribed to treat DM and HTN.

The mean age of the population affected by HTN and DM include 55 years respectively and range of age including 37-80years.

The gender distribution of DM and HTN among females is $52.6 \%$ and males is $47.3 \%$ which shows that gender is nowhere a risk factor.

On an average we manage to get 173 patients medication histories of DM and HTN. Among 173 of them 23patients did not carry their previous prescriptions or medications. By the histories we got to know that Amlodipine and Telmisartan+Hydrochlorthiazide $(14.70 \%)$, are the most prescribed drugs followed by Atenolol (13.7\%), Glimepride+Metformin (11.7\%), Telmisartan (9.8\%), Metformin (9.8\%), Insulin (9.1\%), Glimepride (3.26\%), Voglibose $(3.26 \%)$, chlorthalidone $(2.61 \%)$, Metoprolol (1.96\%), Glibenclamide (1.96\%), Cilnidipine (1.96\%), Spirinolactone+Torsemide $(0.65 \%)$.

\section{DISCUSSION}

In the prospective study performed as the subjects recruited were less in number, the patients with required histories were also less in number. The prevalence of DM and HTN was found to be $29.41 \%$ and $54.5 \%$ respectively in age group of 37-80 years. According to a study the overall prevalence of DM is found to be $16.63 \%$. The prevalence of DM in percentage in different states of India were found to be as follows Maharashtra (39.8\%), Delhi (32.5\%), Tamil Nadu (40.3\%), West Bengal (31.0\%), Karnataka (34.5\%) Andhra Pradesh (37.5\%), Gujarat $(28.9 \%)$ and Madhya Pradesh $(33.7 \%) .{ }^{4}$ The prevalence of type $2 \mathrm{DM}$ has increased from $1.2 \%$ to $11 \%$ over last three decades. $^{4}$

According to Radhakrishnan et al, Several studies are in support of age - specific onset of DM. In this study most of the diabetics were in the age group were between 40 and 60 years. ${ }^{5}$ The world prevalence of diabetes among adults (20-79 years age) will be $6.4 \%$, affecting 285 million adults, in 2010, and may increase to $7.7 \%$, and 439 million adults by 2030 . Between 2010 and 2030, there will be a $69 \%$ increase in numbers of adults with diabetes in developing countries and a $20 \%$ increase in developed countries. ${ }^{7}$ Yagiz uresin et al, conducted a double blind, multicenter trial on 837 patients with DM and HTN and conclude that Combination of aliskiren with ramipril provided great decrease in blood pressure. ${ }^{11-15}$

According to a Review article performed by Anchala R et al, the overall prevalence for hypertension in India was $29.8 \%{ }^{19-22}$ HTN is almost twice as frequent in patients with DM when compared with patients without disease. Conversely, recent data suggest that hypertensive persons are more predisposed to the development of diabetes than are normotensive persons. ${ }^{23-25}$

In terms of compliance, degree of compliance decreases with increasing age, for several reasons. For example, most of the elderly have memory problems related to age, while most of elderly patients have vision and hearing problems that might increase the potential of mistakes in taking medications. ${ }^{26-28}$ Another problem with elderly is that most of them have comorbid conditions and are prescribed with several drugs at the same time which might be confusing to remember. ${ }^{29-31}$

According to Chythra R Rao women were found to be more compliant to Prescription than men, but gender was not identified as a significant factor in other studies..$^{31-33}$

\section{CONCLUSION}

As the prevalence of HTN and DM were ruled out. The study revealed that almost half of the admitted patients were with HTN and DM. The patients are counselled properly to adhere strictly to the prescription. Medication adherence to HTN and DM was found to be good in this study. Since the disease complications were also ruled out, 
the health care professionals are recommended to spread awareness on DM and HTN and disease management in order to control disease and improve health outcomes

Funding: No funding sources

Conflict of interest: None declared

Ethical approval: Not required

\section{REFERENCES}

1. Venugopal K, Mohammed MZ. Prevalence of hypertension in type- 2 diabetes mellitus. CHRISMED J Health Res 2014;1:223-7.

2. Huang XB, Tang WW, Liu Y, Hu R, Ouyang LY, Liu $\mathrm{J}-\mathrm{x}$, et al., Prevalence of diabetes and unrecognized diabetes in hypertensive patients aged 40 to 79 years in southwest China; 2017.

3. Simonson DC, Etiology and prevalence of hypertension in diabetic patients, Diabetes Care (PubMed). 1988 Nov-Dec;11(10):821-7.

4. Shah A, Afzal M, Prevalence of diabetes and hypertension and association with various risk factors among different Muslim populations of Manipur, India, J Diabetes Metab Disord. 2013;12:52.

5. Gupta A, Gupta R, Sarna M, Rastogi S, Gupta VP, Kothari K. Prevalence of diabetes, impaired fasting glucose and insulin resistance syndrome in an urban Indian population. Diabetes research and clinical practice. 2003 Jul 1;61(1):69-76.

6. Fowler MJ. Microvascular and macrovascular complications of diabetes. Clin Diabetes. 2008;26:7782.

7. Long AN, Jack SD, The Comorbidities of Diabetes and Hypertension: Mechanisms and Approach to Target Organ Protection, J Clin Hypertens. 2011 Apr;13(4):244-51.

8. Radhakrishnan S, Ekambaram M. Prevalence of diabetes and hypertension among a tribal population in Tamil Nadu. Arch Med Health Sci. 2015;3:66-71.

9. Gupta A, Gupta R, Sharma KK, Lodha, Achari V, Arthur J, et al. Prevalence of diabetes and cardiovascular risk factors in middle-class urban participants in India, BMJ Open Diabetes Research and Care; 2014.

10. Shaw JE, Sicree RA, Zimmet PZ. Global estimates of the prevalence of diabetes for 2010 and 2030. Diabetes research and clinical practice. 2010 Jan 1;87(1):4-14.

11. Plakas S, Mastrogiannis D, Mantzorou M. Validation of the 8-Item Morisky Medication Adherence Scale in Chronically Ill Ambulatory Patients in Rural Greece. March 2016;6:158-69.

12. Unni EJ, Farris KB. Unintentional non-adherence and belief in medicines in older adults. Patient education and counseling. 2011 May 1;83(2):265-8.

13. Tan XI, Patel I, Chang J. Review of the four item Morisky medication adherence scale (MMAS-4) and eight item Morisky medication adherence scale (MMAS-8). Innovations in Pharmacy. 2014;5(3):5.

14. Uresin Y, Taylor AA, Kilo C, Tschöpe D, Santonastaso M, Ibram G, et al. Efficacy and safety of the direct renin inhibitor aliskiren and ramipril alone or in combination in patients with diabetes and hypertension. Journal of the Renin-AngiotensinAldosterone System. 2007 Dec;8(4):190-200.

15. Konzem SL, Devore VS, Bauer DW, Controlling hypertension in patients with diabetes, Europe PMC., American Family Physician 2002;66(7):1209-14.

16. Gress TW, Nieto FJ, Shahar E, Wofford MR, Brancati FL. Hypertension and antihypertensive therapy as risk factors for type 2 diabetes mellitus. New England Journal of Medicine. 2000 Mar 30;342(13):905-12.

17. Shaw JE, Sicree RA, Zimmet PZ. Global estimates of the prevalence of diabetes for 2010 and 2030. Diabetes Res Clin Pract. 2010;12:4-14.

18. Anchala R, Kannuri KK, Pant H, Khan H, Oscar H, Franco $\mathrm{C}$ et al. Hypertension in India: a systematic review and meta-analysis of prevalence, awareness, and control of hypertension, J Hypertens. 2014 Jun;32(6):1170-7.

19. Osterberg L, Blaschke T. Adherence to medication. N Engl J Med. 2005;353:487-97.

20. Wild S, Roglic G, Green A, Sicree R, King H, Global Prevalence of Diabetes: Estimates for the year 2000 and projections for 2030, Diabetes Care. 2004;27(5):1,047-1,053.

21. Pinhas-Hamiel O, Zeitler P. The global spread of type 2 diabetes mellitus in children and adolescents, J. Pediatr. 2005;146(5):693-700.

22. National High Blood Pressure Education Program Working Group report on hypertension in diabetes, Hypertension. 1994;23(2):145-15.

23. Sowers JR, Epstein M, Frohlich ED. Diabetes, hypertension, and cardiovascular disease: an update, Hypertension. 2001;37(4):1053-9.

24. Praska JL, Kripalani S, Seright AL, Jacobsen TA. Identifying and assisting low-literacy patients with medication use: a survey of community pharmacies. Ann Pharmacother. 2005;39:1441-5.

25. Hill-Briggs F, Gary TL, Bone LR, Hill MN, Levine DM, Bancati FL. Medication adherence and diabetes control in urban African Americans with type 2 diabetes. Health Psychol. 2005;24:349-57.

26. Rubin RR. Adherence to pharmacologic therapy in patients with type 2 diabetes mellitus. Am J Med. 2005;118:27S-34S

27. Grant RW, Singer DE, Meigs JB. Medication adherence before an increase in antihypertensive therapy: a cohort study using pharmacy claims data. Clin Ther. 2005;27:773-81.

28. Pendota S, Surabhineni SAK, Katnapally AS, Porandla D, Beemreddy SK. Classification and applying pharmacovigilance principles to study adverse drug reaction and its management, IJBCP, 2017;6(11).

29. Akshaya Kalyani SS, Srihitha P. An Epidemiological Study on Adverse Drug Reactions in Indian Population: Meta-Analysis. International Journal of Pharmaceutical and Clinical Research. 2017;9(10):654-9. 
30. Sweileh WM, Aker O, Hamooz S. Rate of compliance among patients with diabetes mellitus and hypertension. An-Najah Univ J Research-A-(Natural Sciences). 2005;19:1-12.

31. Al-Mehza AM, Al-Muhailije FA, Khalfan MM, AlYahya AA. Drug compliance among hypertensive patients; an area based study. Eur J Genet Med. 2009;6:6-10.

32. Kumar PN, Halesh LH. Antihypertensive treatment: A study on correlates of non adherence in a tertiary care facility. Int J Biol Med Res. 2010;1:248-52.

33. Rao CR, Kamath VG, Shetty A, Kamath A. Treatment compliance among patients with hypertension and type 2 diabetes mellitus in a coastal population of Southern India. International Journal of Preventive Medicine. 2014 Aug;5(8):992.

Cite this article as: Kalyani SSA, Pendota S, Katnapally S, Porandla D, Bheemreddy S. Cross sectional study on prevalence and medication adherence of hypertension and diabetes in a tertiary care hospital in Karimnagar, India. Int J Basic Clin Pharmacol 2018;7:807-12. 